\title{
REVIEW ARTICLE \\ Chemoprophylaxis and vaccination in preventing subsequent cases of meningococcal disease in household contacts of a case of meningococcal disease: a systematic review
}

\author{
L. TELISINGHE ${ }^{1,2} *$ T. D. WAITE ${ }^{1,3,4}$, M. GOBIN ${ }^{1}$, O. RONVEAUX ${ }^{5}$, \\ K. FERNANDEZ ${ }^{5}$, J. M. STUART ${ }^{2,5,6}$ AND R. J. P. M. SCHOLTEN ${ }^{7}$ \\ ${ }^{1}$ Field Epidemiology Services, Public Health England, Bristol, UK \\ ${ }^{2}$ University of Bristol, Bristol, UK \\ ${ }^{3}$ Field Epidemiology Training Programme, Public Health England, UK \\ ${ }^{4}$ European Programme for Interventional Epidemiology Training (EPIET), European Centre for Disease \\ Prevention and Control (ECDC), Stockholm, Sweden \\ ${ }^{5}$ World Health Organization, Geneva, Switzerland \\ ${ }^{6}$ London School of Hygiene and Tropical Medicine, London, UK \\ ${ }^{7}$ Dutch Cochrane CentrelJulius Center for Health Sciences and Primary Care, University Medical Center \\ Utrecht, Utrecht, The Netherlands
}

Received 15 December 2014; Final revision 10 March 2015; Accepted 1 April 2015;

first published online 28 April 2015

\section{SUMMARY}

Household contacts of an index case of invasive meningococcal disease (IMD) are at increased risk of acquiring disease. In revising WHO guidance on IMD in sub-Saharan Africa, a systematic review was undertaken to assess the effect of chemoprophylaxis and of vaccination in preventing subsequent cases of IMD in household contacts following an index case. A literature search for systematic reviews identified a single suitable review on chemoprophylaxis in 2004 (three studies meta-analysed). A search for primary research papers published since 2004 on chemoprophylaxis and without a date limit on vaccination was therefore undertaken. There were 2381 studies identified of which two additional studies met the inclusion criteria. The summary risk ratio for chemoprophylaxis $v s$. no chemoprophylaxis (four studies) in the 30-day period after a case was $0 \cdot 16[95 \%$ confidence interval (CI) $0 \cdot 04-0 \cdot 64, P=0 \cdot 008]$; the number needed to treat to prevent one subsequent case was $200(95 \%$ CI 111-1000). A single quasi-randomized trial assessed the role of vaccination. The risk ratio for vaccination $v s$. no vaccination at 30 days was $0 \cdot 11(95 \%$ CI $0 \cdot 01-2 \cdot 07, P=0 \cdot 14)$. The results support the use of chemoprophylaxis to prevent subsequent cases of IMD in household contacts of a case. Conclusions about the use of vaccination could not be drawn.

Key words: Infectious disease control, meningococcus ( $N$. meningitis), meningococcal disease, Neisseria meningitides, prevention.

\section{INTRODUCTION}

Invasive meningococcal disease (IMD), caused by Neisseria meningitidis is associated with severe morbidity

\footnotetext{
* Author for correspondence: Dr L. Telisinghe, Field Epidemiology Services, Public Health England, Bristol BS1 6EH, UK.

(Email: lily.telisinghe@phe.gov.uk)
}

and mortality and can result in devastating sequelae $[1,2]$. With the roll out of meningococcal vaccination against the common serotypes through routine immunization programmes and mass prevention campaigns, the incidence of IMD in Western settings and recently in sub-Saharan Africa has fallen markedly [3-6].

Despite these successes gained, sporadic cases and outbreaks of IMD still continue to occur [1]. 
Household contacts of an index case of IMD (defined as the first identified case in a household), are at increased risk of acquiring disease themselves [7, 8]. Primary prevention interventions targeting this high-risk group form the basis of much international guidance, which recommends chemoprophylaxis [9-11], with the aim of eradicating nasopharyngeal carriage. A recent Cochrane review of randomized controlled trials (RCTs) alone did not identify any eligible studies addressing the role of chemoprophylaxis in household contacts [12]. A systematic review by Purcell et al., done nearly a decade ago, of both RCTs and observational studies identified three observational studies which supported the use of chemoprophylaxis in household contacts of an index case of IMD [13].

The role of vaccination in household contacts of an index case of IMD has been debated [14-16]. Given the time required for protective antibodies to develop following vaccination, its role in immediate disease prevention and control strategies in this population, is not completely clear $[14,15]$. There may be benefit from vaccination in addition to chemoprophylaxis, when disease in the index case is caused by a vaccine-preventable strain [16].

As a result of the success of the meningococcal A conjugate vaccine (MenAfriVac) [6] roll out in the African meningitis belt, the World Health Organization (WHO) is updating guidelines on the meningitis outbreak response in sub-Saharan Africa. To inform these guidelines, work was commissioned by the WHO on key areas of the outbreak response, including prevention strategies (chemoprophylaxis and vaccination) in household contacts. We present results of the systematic reviews undertaken to determine (1) the effect of chemoprophylaxis and (2) the effect of vaccination, in preventing cases of IMD in household contacts during the first 30 days and 1 year after an index case.

\section{METHODS}

\section{Search strategy}

Medline, EMBASE, African Index Medicus, Global Index Medicus, Cochrane Database of Systematic Reviews, Cochrane Central Register of Controlled Trials, CAB health and TRIP were searched from inception to December 2013 for original research studies and systematic reviews. A Google search with filters for countries in the African meningitis belt was performed (20022013) for grey literature. Bibliographies of identified studies were searched, and, experts in IMD contacted through the WHO, for additional studies or sources of data. Where possible, authors were contacted for additional information if needed.

The MESH headings and key words used were (1) for chemoprophylaxis: (Chemoprevention or PostExposure Prophylaxis or (chemoprophyl* or chemoprevent* or prophyla*)) and (Anti-Bacterial Agents or Drug Therapy or (antibiotic* or antimicrobial* or antibacterial* or ((drug*) adj3 treatment*) or ((drug*) adj3 therap*)) or (azithromycin* or azithro* or ciprofloxacin* or cipro* or quinol* or fluoroquinol* or fluoro-quinolon* or ceftriaxon* or cefix* or rifampi* or cephalospori*)); (2) for vaccination: Immunization or Vaccination or Meningococcal Vaccines or (vaccin* or immun*); (3) for the outcomes: Neisseria meningitidis or Meningitis, Meningococcal or ('N. meningitid\#s' or meningococ* or ((neisseria or epidemic) adj3 mening*) or ((Neisseria) adj3 disease*) or ((Neisseria) adj3 infect*)); and (4) for the setting: Contact Tracing or Disease Transmission, Infectious or Disease Outbreaks or (contact* or household* or cluster* or transmi* or outbreak* or ((subsequent or associated) adj2 case*). The expressions for each intervention were combined with the outcome and setting using the Boolean operator AND to generate a final output of studies.

\section{Eligibility criteria}

Both (quasi-)RCTs and observational studies with clear intervention and comparator groups were included. The study population was household contacts, defined for this review as persons (adults and children) living and/ or sleeping in the same household/dwelling as an index case of IMD. The included studies had a minimum follow-up period of at least 30 days. The interventions of interest were antibiotics currently recommended as chemoprophylaxis for $N$. meningitidis (e.g. ciprofloxacin, rifampicin, ceftriaxone, azithromycin, cefixime) [9] or those historically used for chemoprophylaxis (e.g. minocycline, sulphonamide) and any vaccine effective against $N$. meningitidis. The comparator groups consisted of those given placebo, no antibiotics or vaccination, or any other intervention. The primary outcome was the occurrence of IMD in household contacts in the 30 days and 1 year following onset of IMD in the index case; henceforth referred to as a subsequent case. Cases occurring in the first $24 \mathrm{~h}$ following the index case were excluded from the analysis as they were not potentially preventable. Studies were included irrespective of language and publication status. 


\section{Study selection, data extraction and risk of bias assessment}

Study selection, data extraction and risk of bias assessments were undertaken by two independent reviewers. Any disagreements were resolved through discussion or, if required, consultation with a third reviewer.

An initial search for systematic reviews was conducted. If an appropriate systematic review was identified, a search for original research studies was conducted from the end date of the review. If a systematic review was not identified, the search was conducted without a date limit. Studies were initially shortlisted based on the title and abstract. Definite selection was based on screening the full texts of shortlisted studies for eligibility. All relevant data were entered onto standardized case report forms. The yield from the search for original research studies was cross-checked with all identified relevant systematic reviews to ensure that all applicable original research studies were captured by the search.

The quality of systematic reviews was assessed using the AMSTAR tool [17]. Minimum criteria assessed were a comprehensive literature search (e.g. completeness of the search string, number of databases searched, restrictions and exclusions, etc.) and duplicate study selection and data extraction. RCTs were assessed for risk of bias using the Cochrane Collaboration Risk of Bias tool [18]. An adapted Newcastle-Ottawa Scale was used [19] (Supplementary Table S1), to comprehensively assess the risk of bias in observational studies.

\section{Statistical analysis}

The results of studies that were deemed clinically homogeneous with respect to the participants, interventions, outcomes and follow-up were combined to generate a summary risk ratio and absolute risk reduction by the use of a fixed-effects model (MantelHaenszel). In studies with no events in either the intervention or comparator groups, $0 \cdot 5$ was added to all four cells of the $2 \times 2$ table. If no events occurred in both the intervention and comparator groups, these studies were not included in the meta-analysis. Statistical heterogeneity was assessed by visual inspection of the forest plot, the $\chi^{2}$ test for homogeneity $(P=0 \cdot 1)$ and the $I^{2}$ statistic. If studies were statistically heterogenous a random-effects model (MantelHaenszel) was used.

All statistical analyses were undertaken using Stata version 12 (Stata Corporation, USA).

\section{RESULTS}

The search for systematic reviews identified two studies with data on the role of chemoprophylaxis in preventing subsequent cases of IMD [9, 13]. Both searched for RCTs and observational studies. The review by Purcell et al. in 2004 identified five studies (four observational studies and a single small RCT), three of which were combined in a meta-analysis [13]. The review undertaken by the European Centre for Disease Prevention and Control (ECDC) in 2010 identified a further observational study since the review by Purcell et al. [9]. When all domains of the AMSTAR tool were compared, the ECDC study relied mainly on one reviewer for study selection and data extraction (J. M. Stuart, personal communication), with fewer databases searched than the review by Purcell et al. Therefore the review by Purcell et al. was used as the starting point of the search for original research studies on the role of chemoprophylaxis $[9,13]$. No systematic reviews were identified on the role of vaccination in preventing subsequent cases of IMD. Therefore the search for original research studies on the role of vaccination was conducted without a date limit.

The search for original research studies identified 2381 studies of which 77 underwent a full text screen (Fig. 1). Of these, two new studies were identified for inclusion in this review, one addressing the role of chemoprophylaxis and the other vaccination. No unpublished data were identified.

\section{Chemoprophylaxis}

A total of six studies met the eligibility criteria [20-25]; the study characteristics are summarized in Table 1. There was one small RCT [20] and five cohort studies [21-25], of which four were suitable for inclusion in the meta-analysis.

Supplementary Tables S2 and S3 present the riskof-bias assessments for the six included studies. All five observational studies used routine treatment decisions specific to the area when interventions were allocated [21-25]. In four studies the intervention groups were representative of household contacts of IMD $[21,22,24,25]$ and the comparator groups were from the same population as the intervention group [21-23, 25]. Baseline characteristics were not provided and comparability between the study groups were not assessed in all studies [21-25]. There was no information for $25-32 \%$ of households followed-up in two studies [22, 23], while information on missing data 


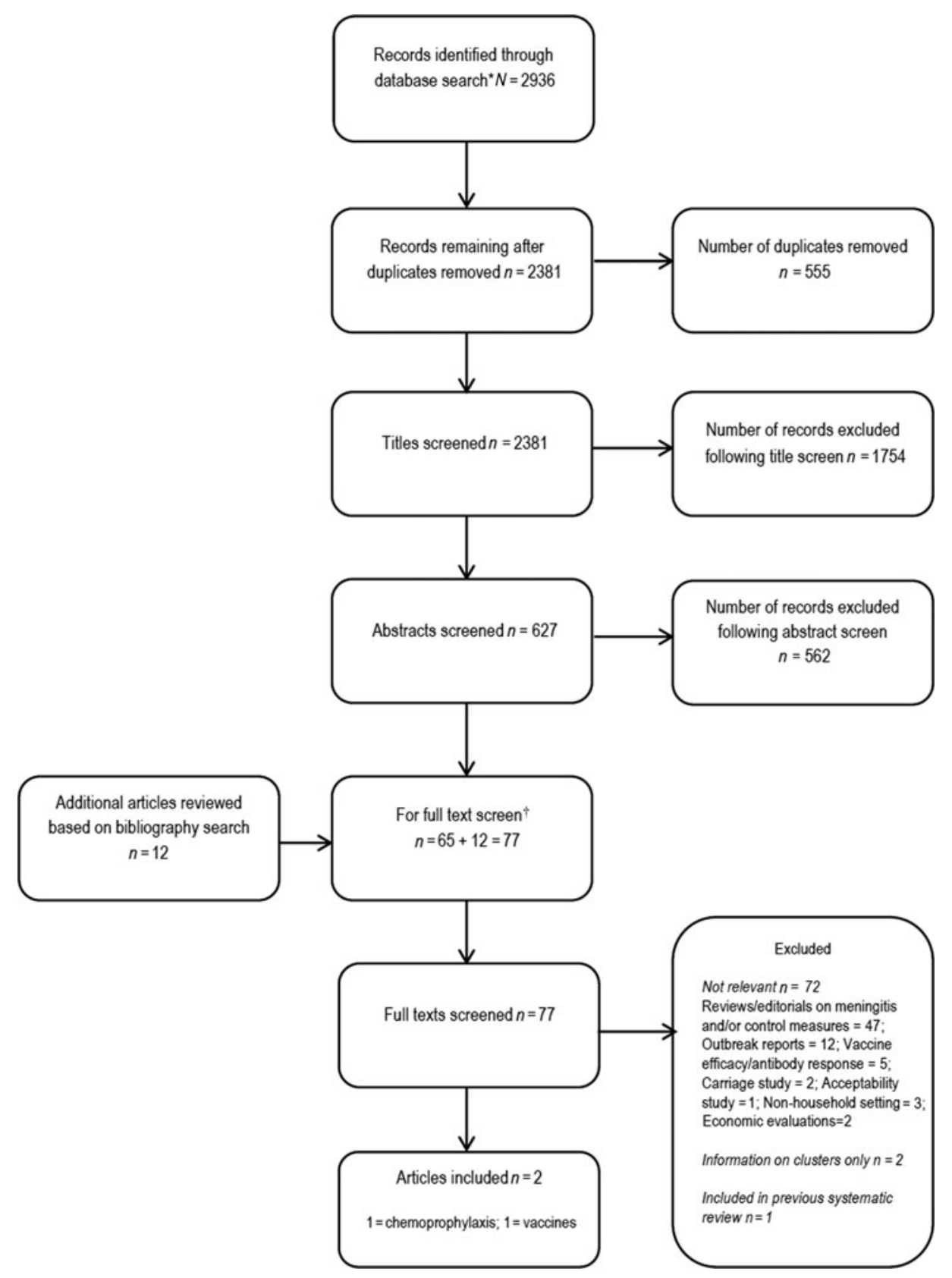

Fig. 1. Study selection process. Flow diagram of number of original research studies considered on the effect of chemoprophylaxis and vaccination in preventing subsequent cases of meningococcal disease in household contacts of a case. * Grey literature included from 2002 onwards. † Forty-five $(63 \cdot 4 \%)$ had no abstract. Chemoprophylaxis: from 2004 onwards; vaccination: no date limit.

was unavailable for the remaining three studies [21, $24,25]$. There was inadequate control for confounding in all studies [21-25].

In the RCT undertaken in Dade County, Florida during an outbreak of IMD, 54 household contacts were randomized to either rifampicin $(n=35)$ or no treatment $(n=19)$ [20]. There was a low to unclear risk of selection bias (while the intervention was allocated by dice throw there was no allocation concealment) and a low risk of performance and detection bias. As there were no subsequent cases in either arms of the trial, this study was not considered further in this review.

An observational study in Telemark, Norway over two time periods, assessed the role of rifampicin given to those harbouring disease-causing strains in addition to standard of care (penicillin to those aged $<15$ years) [24]. No subsequent cases occurred 
in 441 household contacts in the intervention group, compared with 16 subsequent cases in the comparator group [24]. However, as the total number of household contacts in the comparator group was unavailable, a measure of effect could not be calculated and therefore this study was excluded from any further analysis.

The remaining four cohort studies were conducted between 1973 and 2006 in Poland, Denmark, The Netherlands and the USA [21-23, 25]. Disease incidence in the populations were low, ranging from $0 \cdot 23$ to $4 \cdot 00 / 100000$ population. IMD was diagnosed through routine health services. There were 1821 index cases, mostly with IMD caused by serogroups B and C. A total of 5675 household contacts were either given chemoprophylaxis $(n=2322)$ or were in the comparator group $(n=3353)$. Antibiotics used as chemoprophylaxis were rifampicin, ciprofloxacin, minocycline and sulphonamide. The comparator group consisted of those receiving no antibiotics or antibiotics that were deemed unsuitable for use as chemoprophylaxis (e.g. penicillin). The definition of a household contact was similar across studies (Table 1).

Twenty cases of IMD occurred in household contacts of an index case; four in the first $24 \mathrm{~h}$ following the index case and 16 subsequent cases (Supplementary Fig. S1). In the 30 days of follow-up no subsequent cases of IMD occurred in the intervention groups, while 14 subsequent cases occurred in the comparator groups [21-23, 25]. The overall summary risk ratio for the occurrence of subsequent IMD in this 30-day period was $0 \cdot 16[95 \%$ confidence interval $(\mathrm{CI})$ $0 \cdot 04-0 \cdot 64, P=0 \cdot 008]$ in favour of chemoprophylaxis (Fig. 2). The overall summary risk difference was $-0.005(95 \%$ CI -0.009 to $-0.001, P=0.005)$, with the number needed to treat to prevent one subsequent case being 200 (95\% CI 11-1000).

Three studies reported results up to a year of follow-up. In total, two subsequent cases of IMD occurred in the intervention groups, while nine subsequent cases occurred in the comparator groups during the total follow-up period. The overall summary risk ratio and risk difference for the occurrence of subsequent IMD up to a year of follow-up were $0.34 \quad(95 \%$ CI $0 \cdot 11-1 \cdot 06)$ (Supplementary Fig. S2) and $-0.003(95 \% \mathrm{CI}-0.009$ to 0.002$)$, respectively. However, it was unclear if the entire cohort in the included studies were followed up to a year, which would affect the denominators in these calculations.

\section{Vaccination}

A single quasi-RCT conducted in 1977 in Zaria, Nigeria during an epidemic of IMD caused by serogroup A, assessed the effects of vaccination in preventing subsequent cases in 1043 household/close contacts of an index case (Table 1) [26]. The intervention group $(n=520)$ received the Mérieux (meningococcal A and C) vaccine, while the comparator group $(n=523)$ received tetanus toxoid.

Supplementary Table S4 summarizes the risk-of-bias assessment for this trial. There was an unclear risk of selection bias (alternative compounds allocated to intervention and comparator groups, with allocation concealment not being specified), a low risk of performance bias and a low to unclear risk of detection bias (unclear if outcome assessment was blinded, which may have influenced classification of probable cases, but not definite cases).

There were no confirmed subsequent cases of IMD (meningitis with a positive culture, antigen test or rise in antibody titre) in the intervention group, while there were five confirmed subsequent cases in the comparator group. All but one of the confirmed subsequent cases occurred within 30 days of the index case. There was one probable subsequent case of IMD in the intervention group (meningitis with negative cultures and antigen test or septicaemia and high baseline antibody titre or rise in antibody titre) and four probable subsequent cases in the comparator group, all within 30 days of the index case. The risk of subsequent confirmed IMD in the 30-day period in those given meningococcal vaccination was $0 \cdot 11$ (95\% CI $0 \cdot 01-2 \cdot 07, P=0 \cdot 14$ ) times the risk in the comparator group. The risk of subsequent confirmed and probable IMD in the 30-day period in those given meningococcal vaccination was $0.13(95 \% \mathrm{CI}$ $0 \cdot 02-1 \cdot 00, P=0 \cdot 05)$ times the risk in the comparator group.

\section{Selective publication}

Due to the small number of identified studies formal analysis for publication bias could not be undertaken.

\section{DISCUSSION}

This study is the most up to date and complete systematic review of both RCTs and observational studies, on the use of chemoprophylaxis and vaccination in preventing subsequent cases of IMD in household 
Table 1. Characteristics of studies identified on the role of chemoprophylaxis and vaccination in preventing subsequent cases of meningococcal disease in household contacts of a case of meningococcal disease

\begin{tabular}{|c|c|c|c|c|c|c|c|c|c|c|c|c|c|}
\hline $\begin{array}{l}\text { 1st author [ref.], } \\
\text { Design }\end{array}$ & Setting; country; region & Date & $\begin{array}{l}\text { Index } \\
\operatorname{cases}(N)\end{array}$ & $\begin{array}{l}\text { Serogp } \\
\text { of cases }\end{array}$ & $\begin{array}{l}\text { Household contact } \\
\text { definition }\end{array}$ & $\begin{array}{l}\text { No. of } \\
\text { contacts }\end{array}$ & $\begin{array}{l}\text { Total } \\
\text { follow-up } \\
\text { time }\end{array}$ & Intervention & Comparator & $\begin{array}{l}\text { Exposed, } \\
n\end{array}$ & $\begin{array}{l}\text { Exposed } \\
\text { subsequent } \\
\text { cases, } n\end{array}$ & Un-exposed, & $\begin{array}{l}\text { Unexposed } \\
\text { subsequent } \\
\text { cases, } n\end{array}$ \\
\hline \multicolumn{14}{|l|}{ Chemoprophylaxis } \\
\hline $\begin{array}{l}\text { Stefanoff* } 2008 \\
\text { [21], cohort }\end{array}$ & $\begin{array}{l}\text { Endemic; Poland; } \\
\text { National (surveillance } \\
\text { data) }\end{array}$ & $\begin{array}{l}2003 \text { to } \\
2006\end{array}$ & 635 & - & $\begin{array}{l}\text { Person living in the } \\
\text { same } \mathrm{HH} \text { as the } \\
\text { case in the } 7 \text { days } \\
\text { before onset of } \\
\text { illness in the case }\end{array}$ & 1905 & $\begin{array}{l}\text { at least } \\
2 \text { months }\end{array}$ & Rifampicin & No abx & 629 & 1, day 42 & 1276 & 3 , days $6,7,8$ \\
\hline $\begin{array}{l}\text { Samuelsson } 2000 \\
\text { [23], cohort }\end{array}$ & $\begin{array}{l}\text { Endemic }(3-4 / 100000) \\
\text { Denmark; National } \\
\text { (surveillance data) }\end{array}$ & $\begin{array}{l}\text { Oct. } 1995 \text { to } \\
\text { Apr. } 1997\end{array}$ & 172 & - & $\begin{array}{l}\text { Person sleeping in } \\
\text { the same HH/ } \\
\text { room or kissing/ } \\
\text { saliva exchanging } \\
\text { contact with the } \\
\text { case in the } 10 \text { days } \\
\text { before onset of } \\
\text { illness in the case }\end{array}$ & 802 & $>24 \mathrm{~h}$ & Ciprofloxacin & No abx & 724 & 0 & 72 & 2 , days 2,3 \\
\hline $\begin{array}{l}\text { Scholten } 1993 \text { [22], } \\
\text { cohort }\end{array}$ & $\begin{array}{l}\text { Endemic (4/100 000), } \\
\text { Netherlands; National } \\
\text { (surveillance data) }\end{array}$ & $\begin{array}{l}\text { Apr. } 1989 \\
\text { to Apr. } \\
1990\end{array}$ & 502 & $\begin{array}{l}\text { Mostly B, } \\
\text { C, A }\end{array}$ & $\begin{array}{l}\text { Person living in the } \\
\text { same house as the } \\
\text { case in the week } \\
\text { before } \\
\text { hospitalization of } \\
\text { the case }\end{array}$ & 1102 & $\begin{array}{l}\text { At least } 30 \\
\text { days }\end{array}$ & $\begin{array}{l}\text { Rifampicin or } \\
\text { minocycline }\end{array}$ & $\begin{array}{l}\text { No abx or abx } \\
\text { other than } \\
\text { rifampicin or } \\
\text { minocycline }\end{array}$ & 276 & 1 , day 35 & 826 & $\begin{array}{l}4 \text {, days } 2,4 \text {, } \\
4,13\end{array}$ \\
\hline $\begin{array}{r}\text { Kristiansen } 1992 \\
\text { [24], time series }\end{array}$ & $\begin{array}{l}\text { Endemic (1986: 6.7/100 } \\
\text { 000); Norway; Telemark } \\
\text { (surveillance data) }\end{array}$ & $\begin{array}{c}\text { Jan. } 1984 \text { to } \\
\text { Dec. } 1989\end{array}$ & 13 & $\begin{array}{l}8 \mathrm{~B}, 4 \mathrm{C} \\
1 \mathrm{Y}\end{array}$ & - & $\begin{array}{l}441 \text { (during } \\
1987-1989 \text { ) }\end{array}$ & - & $\begin{array}{l}\text { Rifampicin if } \\
\text { harbouring } \\
\text { disease } \\
\text { causing strain } \\
\text { + penicillin in } \\
<15 \text { years } \\
\text { (1987-1989) }\end{array}$ & $\begin{array}{l}\text { Penicillin if } \\
<15 \text { years only } \\
(1984-1987)\end{array}$ & 441 & 0 & - & $16 \dagger$ \\
\hline $\begin{array}{l}\text { MDSG } 1976 \text { [25], } \\
\text { cohort }\end{array}$ & $\begin{array}{l}\text { Endemic }(0 \cdot 23 / 100000) \\
\text { USA; } 27 \text { states and } \\
\text { Washington DC in } \\
\text { period } 1 ; 17 \text { states and } \\
\text { Washington DC in } \\
\text { period } 2\end{array}$ & $\begin{array}{l}\text { Nov. } 1973 \\
\text { to Mar. } \\
\text { 1974; } \\
\text { Jan.-Apr. } \\
1975\end{array}$ & $\begin{array}{r}512(324 \\
\text { serogp) }\end{array}$ & $\begin{array}{l}45 \% \mathrm{~B}, 32 \% \\
\mathrm{C}, 18 \% \mathrm{Y} \\
2 \% \mathrm{~A}\end{array}$ & $\begin{array}{l}\text { Person that lived in } \\
\text { the same HH/ } \\
\text { dormitory room } \\
\text { with a case in the } \\
\text { week prior to onset } \\
\text { of illness in the } \\
\text { case }\end{array}$ & 1872 & 30 days & $\begin{array}{l}\text { Rifampicin, } \\
\text { sulphonamide } \\
\text { or minocycline }\end{array}$ & $\begin{array}{l}\text { No abx or abx } \\
\text { other than } \\
\text { sulfonamide, } \\
\text { minocycline } \\
\text { or rifampicin }\end{array}$ & 693 & 0 & 1179 & $\begin{array}{l}5 \text {, days } 10 \\
11,12 \\
14,27\end{array}$ \\
\hline $\begin{array}{l}\text { Kaiser } 1974[20], \\
\text { randomized trial }\end{array}$ & $\begin{array}{l}\text { Outbreak USA; Dade } \\
\text { County, Florida }\end{array}$ & $\begin{array}{l}\text { Apr--Dec. } \\
1970\end{array}$ & n.a. & $\mathrm{C}$ & $\begin{array}{l}\text { People who slept/ate } \\
\text { in the same } \\
\text { dwelling as the } \\
\text { case }\end{array}$ & & 9 months & Rifampicin & None specified & 35 & 0 & 19 & 0 \\
\hline
\end{tabular}




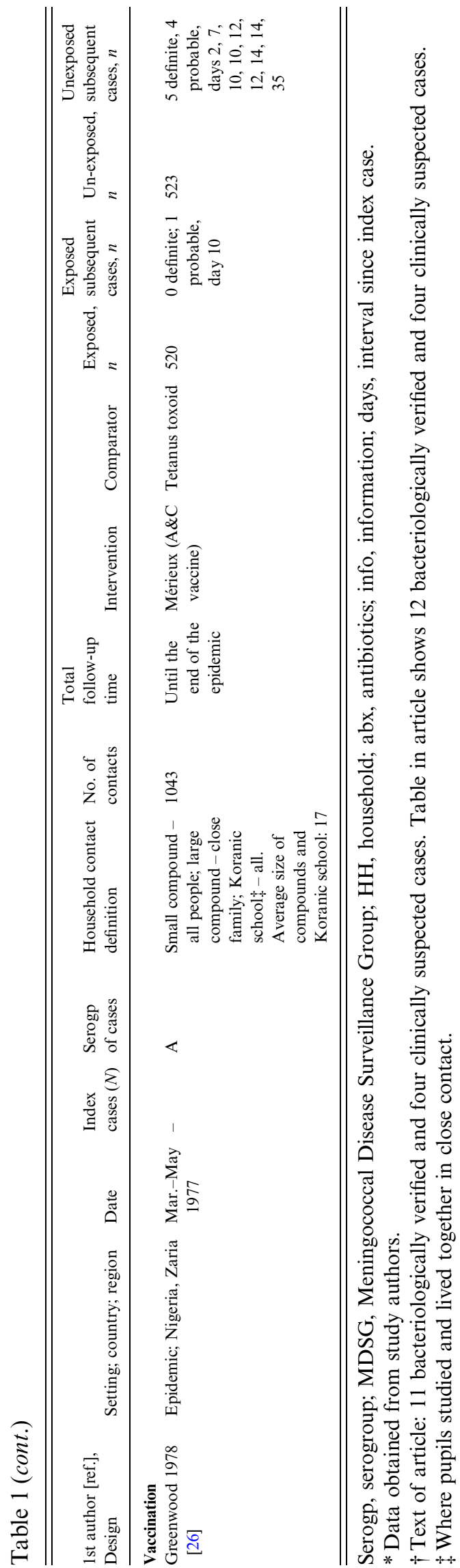

contacts. Based on four observational studies an $84 \%$ reduction in the risk of subsequent IMD in the 30-day period after an index case was found in household contacts given chemoprophylaxis. However, the absolute risk reduction was small, with 200 household contacts needing treatment with chemoprophylaxis to prevent one subsequent case. No statistically significant difference in the risk of confirmed IMD was found after 30 days in household contacts given meningococcal vaccination compared to those given tetanus-toxoid vaccine.

Our results update and support the findings of the previous systematic review by Purcell et al. [13]. We found an additional observational study on the role of chemoprophylaxis in household contacts published since 2004 [21]. This study was the same additional study identified by the ECDC review [9]. All four studies combined to generate a summary effect estimate were cohort studies [21-23, 25], with serious limitations which need to be considered when interpreting results. Information on baseline characteristics of the intervention and comparator groups were not available. While adjustment for risk factors (such as age, socioeconomic status) would not have been possible in individual studies given the small number of subsequent cases, the lack of baseline risk factor information leaves the comparability of the two groups in doubt. Imbalances in baseline characteristics could in part have explained the results seen. Data on the completeness of follow-up in the two groups were not provided; therefore it is not possible to consider selection bias in the included studies. However, most studies used routine national surveillance data and therefore differential follow-up in the groups is unlikely. There were a large proportion of missing results in two studies where data was provided, raising concerns about the generalizability of findings. The small number of subsequent cases is reflected in the width of the confidence intervals for the measures of effect, resulting in imprecise summary estimates. Notwithstanding these limitations and the small number of studies, there was consistency in findings across all included studies. This, combined with the biological plausibility of the intervention and the severity of IMD, adds weight to our findings and lends support to the use of chemoprophylaxis in household contacts of an index case of IMD.

Studies available for inclusion in this review on chemoprophylaxis were all from endemic countries in Europe and the USA in non-outbreak settings. Therefore the applicability of the results to settings in sub-Saharan Africa is uncertain. WHO guidance, 


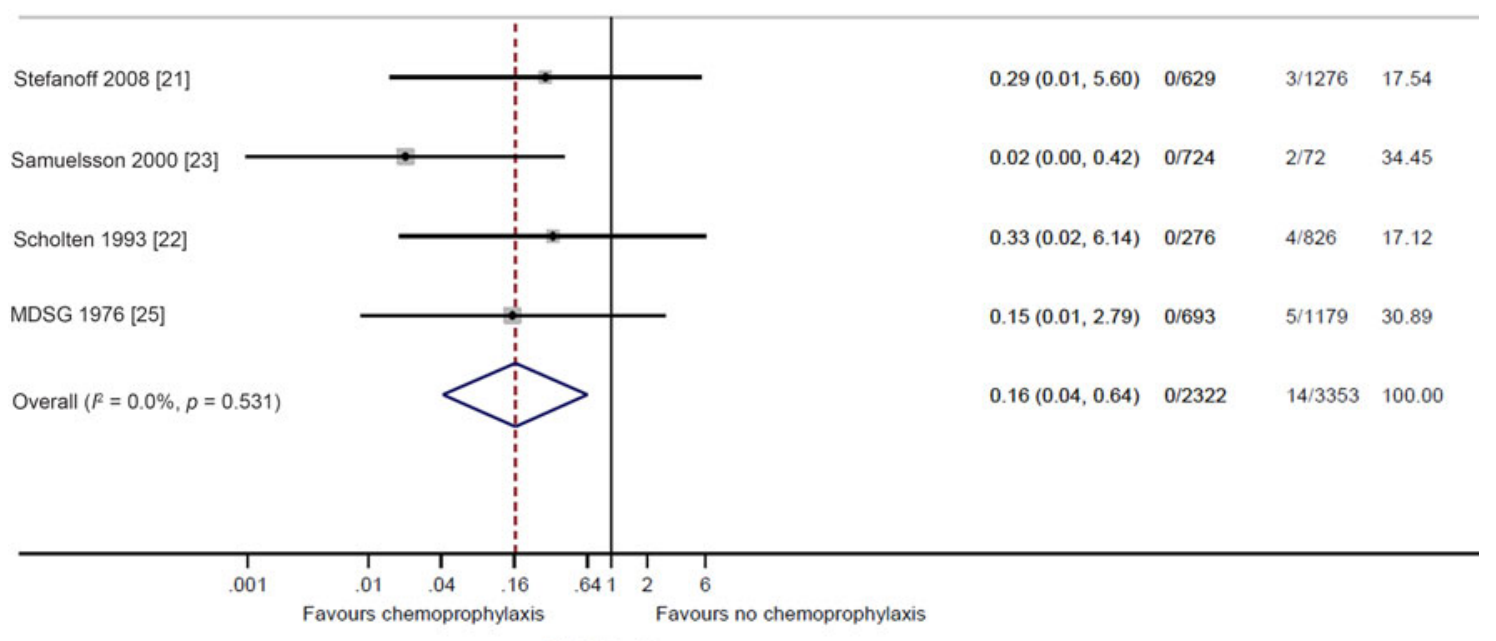

Risk Ratio

Fig. 2. Forest plot of the risk of subsequent cases of meningococcal disease in the 30 days after an index case in household contacts given and not given chemoprophylaxis.

unchanged after this review, is that chemoprophylaxis is recommended for household contacts of an index case of IMD in non-epidemic periods but not during epidemics [27].

Studies have demonstrated that the risk of IMD to household contacts is highest in the first week following the index case, and rapidly declines thereafter [7, 28]. Our findings of a decreased risk of subsequent cases in the 30 days after chemoprophylaxis therefore support its use during this period of risk. While the data suggested a $66 \%$ reduction in the risk of subsequent IMD up to a year of follow-up, there was insufficient information regarding the follow-up data to draw firm conclusions on the sustained effects of chemoprophylaxis following the first 30 days.

Ciprofloxacin and rifampicin are two commonly used chemoprophylactic agents, as they are known to be effective in eradicating nasopharyngeal carriage of $N$. meningitidis, can be given orally and are associated with generally mild and similar side-effect profiles [12]. Antibiotic resistance is known to develop in meningococcal isolates exposed to rifampicin, while this is not seen to occur with ciprofloxacin [12]. In addition, in sub-Saharan Africa fears of tuberculosis resistance to rifampicin is likely to limit its use as a chemoprophylactic agent. The cost of ciprofloxacin is cheaper than rifampicin (US\$4 per 100 contacts treated vs. US\$48, respectively) [29], with ciprofloxacin being given as a single dose and rifampicin needing a longer duration of treatment [12]. Therefore, ciprofloxacin would be the preferred chemoprophylactic agent [12]. The cost associated with providing chemoprophylaxis to household contacts in the UK showed varying results; following a case of IMD in a primary school pupil it was estimated at $£ 318$ [30], while a modelling study estimated this cost to be $£ 68$ [31]. A study modelling chemoprophylaxis and vaccination to household contacts following a case of IMD in school-aged children in the UK estimated this intervention to be cost saving at high relative risks of subsequent cases (cost per case prevented $-£ 338$ at a relative risk of 700) [32].

A single trial was identified which evaluated the role of vaccination in household contacts in preventing subsequent cases of IMD [26]. While the data suggested an $89 \%$ reduction in the risk of definite subsequent IMD in household contacts given vaccination, there was insufficient evidence to rule out a chance finding $(P=0 \cdot 14)$. Given the dearth of information, conclusions cannot be drawn on the use of vaccination in household contacts. However, it should be noted that European guidance, which has been widely adopted, recommends the use of vaccination in addition to chemoprophylaxis in household contacts, when disease in the index case is caused by a vaccinepreventable strain [9]. 
In conclusion, this systematic review supports the use of chemoprophylaxis in household contacts of a case of IMD to prevent subsequent cases in nonepidemic settings. There is insufficient evidence from this review to draw conclusions about the benefits of vaccination for household contacts.

\section{SUPPLEMENTARY MATERIAL}

For supplementary material accompanying this paper visit http://dx.doi.org/10.1017/S0950268815000849.

\section{ACKNOWLEDGEMENTS}

The authors thank Pawel Stefanoff and Brian Greenwood for additional information regarding their studies. Thanks are also due to Thomas Allen, librarian at the $\mathrm{WHO}$, for guidance on the literature search strategy.

This research received no specific grant from any funding agency, commercial or not-for-profit sectors.

\section{DECLARATION OF INTEREST}

None.

\section{REFERENCES}

1. Rosenstein NE, et al. Meningococcal disease. New England Journal of Medicine 2001; 344: 1378-1388.

2. Borg $\mathbf{J}$, et al. Outcomes of meningococcal disease in adolescence: prospective, matched-cohort study. Pediatrics 2009; 123: e502-509.

3. Trotter CL, et al. Effectiveness of meningococcal serogroup $\mathrm{C}$ conjugate vaccine 4 years after introduction. Lancet 2004; 364: 365-367.

4. Trotter CL, Ramsay ME. Vaccination against meningococcal disease in Europe: review and recommendations for the use of conjugate vaccines. FEMS Microbiology Reviews 2007; 31: 101-107.

5. Trotter CL, et al. A surveillance network for meningococcal disease in Europe. FEMS Microbiology Reviews 2007; 31: 27-36.

6. Daugla DM, et al. Effect of a serogroup A meningococcal conjugate vaccine (PsA-TT) on serogroup A meningococcal meningitis and carriage in Chad: a community study [corrected]. Lancet 2014; 383: 40-47.

7. De Wals $\mathbf{P}$, et al. Meningococcal disease in Belgium. Secondary attack rate among household, day-care nursery and pre-elementary school contacts. Journal of Infection 1981; 3 (1 Suppl): 53-61.

8. Cooke RP, et al. Secondary cases of meningococcal infection among close family and household contacts in England and Wales, 1984-7. British Medical Journal 1989; 298: 555-558.
9. ECDC. Public health management of sporadic cases of invasive meningococcal disease and their contacts. Stockholm: European Centre for Disease prevention and Control, 2010.

10. WHO. Control of epidemic meningococcal disease, WHO practical gudielines. World Health Organization, Geneva, 1998.

11. CDC. Manual for the Surveillance of Vaccine-Preventable Diseases. Centers for Disease Control and Prevention, USA, 2011.

12. Zalmanovici Trestioreanu A, et al. Antibiotics for preventing meningococcal infections. Cochrane Database of Systematic Reviews 2013; 10: CD004785.

13. Purcell B, et al. Effectiveness of antibiotics in preventing meningococcal disease after a case: systematic review. British Medical Journal 2004; 328: 1339.

14. Artenstein MS. Prophylaxis for meningococcal disease. Journal of the American Medical Association 1975; 231: 1035-1037.

15. Gardner P. Clinical practice. Prevention of meningococcal disease. New England Journal of Medicine 2006; 355: 1466-1473.

16. Hoek MR, et al. Effectiveness of vaccinating household contacts in addition to chemoprophylaxis after a case of meningococcal disease: a systematic review. Epidemiology and Infection 2008; 136: 1441-1447.

17. Shea BJ, et al. Development of AMSTAR: a measurement tool to assess the methodological quality of systematic reviews. BMC Medical Research Methodology 2007; 7: 10.

18. Higgins JPT, Altman DG, Sterne JAC. Chapter 8: Assessing risk of bias in included studies. In: Higgins JPT, Green S, eds. Cochrane Handbook for Systematic Reviews of Interventions, version 5.1.0 (updated March 2011). (www.cochrane-handbook.org).

19. Wells GA, et al. The Newcastle-Ottawa Scale (NOS) for assessing the quality of nonrandomised studies in meta-analyses. (http://www.ohri.ca/programs/clinical_ epidemiology/oxford.htm).

20. Kaiser AB, et al. Seroepidemiology and chemoprophylaxis disease due to sulfonamide-resistant Neisseria meningitidis in a civillian population. Journal of Infectious Diseases 1974; 130: 217-224.

21. Stefanoff $\mathbf{P}$, et al. The detection of meningococcal household clusters and their prophylaxis in the changing epidemiological situation of invasive meningococcal disease in Poland, 2003-2006. Eurosurveillance 2008; 13(10).

22. Scholten RJ, et al. Secondary cases of meningococcal disease in The Netherlands, 1989-1990; a reappraisal of chemoprophylaxis [in Dutch]. Nederlands Tijdschrift voor Geneeskunde 1993; 137: 1505-1508.

23. Samuelsson S, et al. Prevention of secondary cases of meningococcal disease in Denmark. Epidemiology and Infection 2000; 124: 433-440.

24. Kristiansen BE, et al. Preventing secondary cases of meningococcal disease by identifying and eradicating disease-causing strains in close contacts of patients. Scandinavian Journal of Infectious Diseases 1992; 24: 165-173. 
25. Meningococcal Disease Surveillance Group. Analysis of endemic meningococcal disease by serogroup and evaluation of chemoprophylaxis. Journal of Infectious Diseases 1976; 134: 201-204.

26. Greenwood BM, Hassan-King M, Whittle HC. Prevention of secondary cases of meningococcal disease in household contacts by vaccination. British Medical Journal 1978; 1: 1317-1319.

27. WHO. Meningitis outbreak response in sub-Saharan Africa. World Health Organization, 2014 (http://apps. who.int/iris/bitstream/10665/144727/1/WHO_HSE_PED_ CED_14.5_eng.pdf?ua=1).

28. Hastings L, et al. A retrospective survey of clusters of meningococcal disease in England and Wales, 1993 to 1995: estimated risks of further cases in household and educational settings. Communicable Disease Report. CDR Review 1997; 7: R195-200.

29. Management Sciences for Health. International Drug Price Indicator Guide, Management Sciences for Health, 2012.

30. Letouze D, Yao G, Clarke SC. The costs associated with the public health management of a cluster of meningococcal infection in England. Vaccine 2014; 32: 5549-5551.

31. Christensen $\mathbf{H}$, et al. Introducing vaccination against serogroup B meningococcal disease: an economic and mathematical modelling study of potential impact. Vaccine 2013; 31: 2638-2646.

32. Round A, Palmer S. Should we be doing more to prevent Group $\mathrm{C}$ meningococcal infection in school age children? How can we decide? Journal of Public Health Medicine 1999; 21: 8-13. 